\title{
SAR Image Stacking for the Exploitation of long-term coherent Targets
}

\author{
Francesco De Zan and Paco López-Dekker
}

\begin{abstract}
This paper shows that in a repeat-pass dataset of SAR images a long-term coherent component, when present, can be recovered by coherent temporal filtering of the SAR images and can successively form interferograms with higher SNR. The validity of the idea is confirmed through simulations and one example with real TerraSAR-X data. The theoretical necessity of using long-term interferograms is also discussed and linked to autoregressive processes, starting from the observation that the optimal weighting is given by the inverse of the covariance matrix.
\end{abstract}

Index Terms-SAR interferometry

\section{INTRODUCTION}

Future SAR satellite missions will produce large datasets of interferometric data with unprecedented characteristics. Along with large coverages they will feature short repeat-pass times and medium to high resolution. Tight orbit control and large bandwidths will allow us to neglect geometric (spectral shift) and volumetric decorrelation, so that the main role is going to be played by temporal decorrelation and system noise (thermal noise, quantization, ambiguities...).

Theoretical investigations in recent years have clarified the interferometric performances when the targets are decorrelating (Cramér-Rao bound) and produced an optimal estimator that performs closely to the bound [1] for an arbitrary coherence scenario. It involves the computation of all possible interferograms and a non-linear optimization. However the required computational effort is large and more agile solutions might be needed for processing the data of missions that aim at a global coverage.

This letter discusses a special coherence scenario, in which the presence of long-term coherent components enables a simplified processing, not requiring the simultaneous generation of all the interferograms and still performing close to the bound. Our investigation tries also to shed light on the nature of some coherent stacks, for which the most coherent interferometric pairs are not necessarily those who carry most of the information.

There are cases when the valuable information is paradoxically given by pairs with large temporal separation, despite their low coherence. This is what happens when the target is a superposition of a long-term coherent component and a short time coherent component. The main purpose of this paper is to introduce a novel way to filter the long-term coherent component and apply interferometric tools to it.

The authors are with the Microwaves and Radar Institute of DLR (German Aerospace Center), P.O. Box 1116, D-82230 Wessling, Germany. email:francesco.dezan@dlr.de

\section{INTERFEROMETRIC PROCESSING}

We want to follow the path laid down in [2] and [1], and we adopt a similar notation to those papers. We consider a stack of $N$ images, which have been focused and coregistered to a common reference. The complex value of the pixel of image $n$ for pixel $l$ will be indicated by $y_{n}(l)$. We address distributed targets (surface or volume) which can be modeled as circular gaussian random variables and completely characterized by their coherences $\gamma_{n, m}$ plus their powers $\sigma_{n}^{2}$. For convenience we introduce also the $N \times N$ matrix $\Gamma$ such that

$$
\{\boldsymbol{\Gamma}\}_{n, m}=\gamma_{n, m} .
$$

To fully describe interferograms we need also the interferometric phase which will be indicated by $\phi_{n}$. What the interferograms actually give are noisy estimates of the phase differences $\phi_{n}-\phi_{m}$. To summarize, the expected value of the interferogram will be:

$$
\mathrm{E}\left[y_{n}(l) y_{m}(l)^{*}\right]=\gamma_{n, m} \sigma_{n} \sigma_{m} \exp \left(j\left(\phi_{n}-\phi_{m}\right)\right),
$$

where it is understood that coherence, powers, and propagation phases are considered constant within a neighborhood and dependent only on the image pair. The multilooked interferogram will be:

$$
\left\{\mathbf{I}_{\Omega}\right\}_{n, m}=\sum_{l=1}^{L} y_{n}(l) y_{m}(l)^{*}
$$

for a neighborhood or window of $L$ looks.

In [2], [1] the Cramér-Rao bound (CRB) for the estimation of the $N$ propagation phases $\phi_{n}$ is derived, given a certain coherence scenario described by $\Gamma$ and $L$ looks. These phases will include atmospheric delays (ionosphere, troposphere), orbital errors, uncompensated topographic terms and deformation terms. At this point we are not discussing how to optimally separate the various terms, which is the next logical step. In the same paper [1] the authors suggest also an estimator that performs very close to the CRB. This estimator requires the use of all multilooked interferograms $(N(N-1) / 2)$. For a stack of 200 images the interferogram matrix will have 40,000 entries. For this reason the computational burden is very large and there is a considerable interest in reducing it while maintaining the performance.

\section{SHORT TEMPORAL BASELINES}

In order to reduce the computational effort, one could think of using only a subset of all the interferograms. Intuitively, the most straightforward idea is to use the most coherent interferograms, since - taken individually - they are the ones 
that give the best phases. For example, this is the philosophy of the SBAS technique as presented in [3]. However as it was shown in [1], [4], there are relevant coherence scenarios when this approach can yield large errors (compared to the CRB), especially between the beginning and the end of the phase history.

For a uniform sampling of the time axis and a stationary coherence scenario, the use of lag-1 interferograms, i.e. the elements $\left\{\mathbf{I}_{\Omega}\right\}_{n, n+1}$, is only optimal in the case of purely exponential decorrelation:

$$
\gamma_{n, m}=\exp (-|n-m| / \tau)
$$

where $\tau$ is the time constant of the exponential decay and is normalized to the repeat-pass time.

This can be shown considering that the optimal weighting is given by the inverse of the coherence matrix $\Gamma^{-1}$ [5], [1] and such a matrix is tridiagonal if and only if the direct can be expressed as a pure exponential decay.

It is possible to generalize this reasoning to banded matrices. The limitation to a certain time lag $K$ (i.e. the exclusive use of the $\left\{\mathbf{I}_{\Omega}\right\}_{n, m}$ for $|n-m| \leq K$, justified by a $2 K+1$ banded $\Gamma^{-1}$ ) is related to the possibility of modeling the underlying scatter signal as an autoregressive process of order $K$ [6]. Such a modeling is not possible for targets that exhibit a coherence plateau at large temporal baselines (without taking a large number of terms of course).

Intuitively the idea is that the existence of persisting longterm coherent components make interferograms with large temporal baseline very valuable. These components need not to be particularly strong, for example they could account for 10 or $20 \%$ of the total power, so that the residual coherence would be only 0.1 or 0.2 . This coherence scenario seem to be probable in L-band, according to studies reported in [7] and [8].

\section{COHERENT SYNTHESIS OF VIRTUAL IMAGES}

The purpose of this section is to show how to filter a longterm coherent component, at the beginning and at the end of the stack, and produce high SNR interferograms. Later, section VI discusses the conditions that make this filtering possible.

From the whole stack of images (ordered temporally) one selects a subset of $S$ images positioned at the beginning. For example, from a stack of 200 images one selects the first 20 . Then one estimates the $S$ optimum phases that correspond to that subset, for example using the algorithm found in [1] or some simplified version, depending on the coherence type. Let us call them $\phi_{1}^{1}, \ldots, \phi_{S}^{1}$. These phases allow us to coherently filter the stack subset and produce a single virtual image $v_{1}$ that "represents" the $S$ images at the beginning of the stack:

$$
v_{1}(l)=(1 / S) \sum_{n=1}^{S} y_{n}(l) \exp \left(-j \phi_{n}^{1}\right)
$$

Exactly the same thing can be repeated on the images at the end of the stack, producing a second image $v_{2}$. This process is to be performed on a window basis, since the estimation of the $\phi_{n}$ can be valid only locally.
TABLE I

THE PERFORMANCE OF THE PROPOSED PHASE ESTIMATOR USING 60+60 IMAGES OUT OF A STACK OF 200.

\begin{tabular}{|l|c|}
\hline \hline$N$ (dataset size) & 200 \\
\hline$S$ (subset size) & 60 \\
\hline$L$ (looks) & 100 \\
\hline$\gamma_{\infty}$ & 0.2 \\
\hline$\gamma_{0}$ & 0.8 \\
\hline$\tau$ & 3 \\
\hline measured $\gamma_{v}$ & 0.75 \\
\hline$\gamma_{v}$ from $(7)$ & 0.77 \\
\hline $\mathrm{CRB} \operatorname{std}\left(\phi_{N}-\phi_{1}\right)$ & $0.174 \mathrm{rad}$ \\
\hline $\operatorname{std}\left(\phi_{N}-\phi_{1}\right)$ & $0.186 \mathrm{rad}$ \\
\hline $\operatorname{loss}$ & $0.6 \mathrm{~dB}$ \\
\hline \hline
\end{tabular}

Finally one can build the interferogram between the two virtual images and multilook it:

$$
I_{1,2}=\sum_{l=1}^{L} v_{1}(l) v_{2}^{*}(l)
$$

This equation contains implicitely all the interferograms between the $S$ images in the first subset and the $S$ images in the second subset. The coherence between the two virtual images is approximately given by the following expression:

$$
\gamma_{v}=\frac{\bar{\gamma}_{1,2}}{\sqrt{\bar{\gamma}_{1,1} \bar{\gamma}_{2,2}}}
$$

where

$$
\begin{aligned}
\bar{\gamma}_{1,1} & =\sum_{1 \leq n \leq S} \sum_{1 \leq m \leq S} \gamma_{n, m} \\
\bar{\gamma}_{1,2} & =\sum_{1 \leq n \leq S} \sum_{N-S<m \leq N} \gamma_{n, m} \\
\bar{\gamma}_{2,2} & =\sum_{N-S<n \leq N} \sum_{N-S<m \leq N} \gamma_{n, m}
\end{aligned}
$$

and assuming the re-phasing was good enough.

The coherence $\gamma_{v}$ is directly proportional to $\bar{\gamma}_{1,2}$ and inversely to $\bar{\gamma}_{1,1}$ and $\bar{\gamma}_{2,2}$. Intuitively this means that we need a lasting coherent component, and enough decay within the substacks so that it can be recovered. From the point of view virtual interferograms the short-lived components are just like noise and should be suppressed.

The actual performance for the estimation of a phase difference between two specific images (from the two substacks) is worse than the one for the two virtual images, since we have to consider the uncertainty on the determination of the phases within each sub-stack.

\section{Simulations}

We simulated complex-gaussian data according to the following coherence law (for $n \neq m$ ):

$$
\gamma_{n, m}=\left(\gamma_{0}-\gamma_{\infty}\right) \exp (-|n-m| / \tau)+\gamma_{\infty} .
$$

It represents an exponential decay from $\gamma_{0}$ to $\gamma_{\infty} \neq 0$ with a time constant $\tau$. The time constant is normalized to the repeatpass time and we assume a uniform sampling of the temporal axis. 
TABLE II

THE PERFORMANCE OF THE PROPOSED PHASE ESTIMATOR USING 30+30 IMAGES OUT OF A STACK OF 200.

\begin{tabular}{|l|c|}
\hline \hline$N$ (dataset size) & 200 \\
\hline$S$ (subset size) & 30 \\
\hline$L$ (looks) & 100 \\
\hline$\gamma_{\infty}$ & 0.2 \\
\hline$\gamma_{0}$ & 0.8 \\
\hline$\tau$ & 3 \\
\hline measured $\gamma_{v}$ & 0.62 \\
\hline$\gamma_{v}$ from $(7)$ & 0.63 \\
\hline CRB std $\left(\phi_{N}-\phi_{1}\right)$ & $0.174 \mathrm{rad}$ \\
\hline $\operatorname{std}\left(\phi_{N}-\phi_{1}\right)$ & $0.194 \mathrm{rad}$ \\
\hline $\operatorname{loss}$ & $1.0 \mathrm{~dB}$ \\
\hline \hline
\end{tabular}

Tables I and II report the results of 1000 simulations, together with the selected parameters of the coherence law. In these examples the decay is so fast that the coherence between any of the first images and any of the last ones is practically $\gamma_{\infty}=0.2$. However the coherence of the virtual images is 0.75 or 0.62 depending on the dimension of the sub-stacks $(S)$. Such coherence is well predicted by (7).

The loss on the variance of the phase difference between the first and the last image in the stack $\left(\phi_{N}-\phi_{1}\right)$ is also limited to $0.6-1 \mathrm{~dB}$. This has to be compared with something like 5-7 dB ([4], [9]) when one uses only the most coherent interferograms (between one image and the next in the time series).

\section{DISCUSSION}

The simulations show something that is counter-intuitive at first glance: stacks which are almost decorrelated can hide an interferometric potential. This work shows also that the gains obtainable by the algorithm in [1] are not due to the simple chaining of highly coherent interferograms, but to something completely different. The interpretation in the realm of SAR images is easier: the coherent integration within the sub-stacks attenuates both noise and short-lived components, which act like noise for those long-temporal-baseline interferograms.

Of course for the proposed procedure to work, it is essential that some coherent component exists between the two subsets. When this is not the case, the interferogram between the synthetized images will be decorrelated and no information will be retrieved.

The importance of getting a low variance between the extremes of the time series is clear, one example is the case of the estimation of deformation velocities, since the errors in the average velocity are linearly scaled with the temporal interval over which the total motion is estimated. The problem is related to what is discussed in [10] about delay estimation.

The proposed technique is similar to Persistent Scatterer Interferometry (PSI) ([11]) in that we are interested in phase measures between distant dates. However PSI requires a high SNR on each pixel it considers, whereas here we are not requiring a particular phase or amplitude stability. The signal of interest could be in fact almost buried into the noise, if it is really there we should be able to extract it thanks to multilooking and stacking.

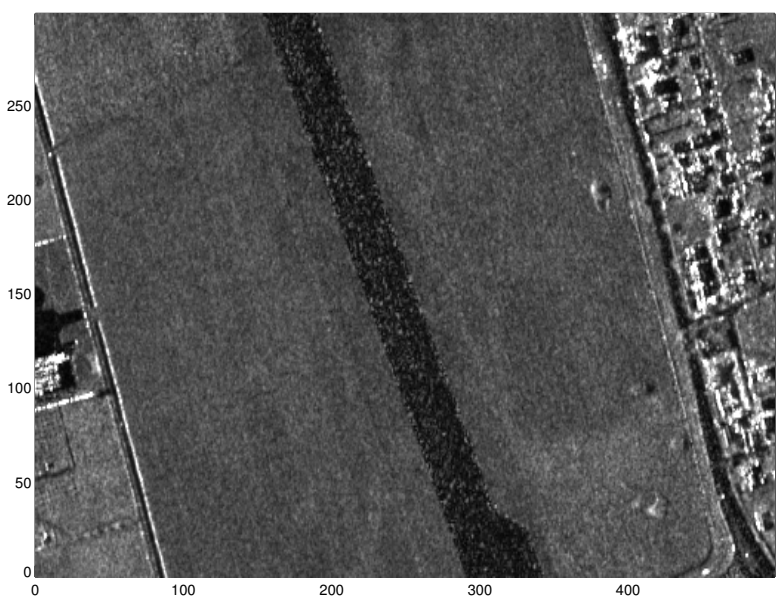

Fig. 1. Mean intensity image of Crotone (Italy) airport runway (TerraSAR-X, (C)DLR 2008-2010).

From another point of view this work is related to the polarimetric-interferometric coherence optimization proposed by Cloude and Papathanassiou in [12] and successively extended by Neumann and co-authors in [13] to multibaseline datasets. In (5) we implicitly suggest to use equal weights for all the images, however the knowledge of the coherence matrix $\Gamma$ would enable the determination of optimal weights (with some additional computational cost), analogously to what was proposed in [12] for polarimetric interferometry. The optimal weights are those that maximize the coherence $\gamma_{v}$ between the two virtual images and can be determined solving the following eigenvalue problems and choosing the eigenvectors $\mathbf{w}_{1}$ and $\mathbf{w}_{2}$ corresponding to the highest eigenvalues:

$$
\begin{aligned}
& \boldsymbol{\Gamma}_{11}^{-1} \boldsymbol{\Gamma}_{12} \boldsymbol{\Gamma}_{22}^{-1} \boldsymbol{\Gamma}_{12}^{T} \mathbf{w}_{1}=\lambda_{1} \mathbf{w}_{1} \\
& \boldsymbol{\Gamma}_{22}^{-1} \boldsymbol{\Gamma}_{12}^{T} \boldsymbol{\Gamma}_{11}^{-1} \boldsymbol{\Gamma}_{12} \mathbf{w}_{2}=\lambda_{2} \mathbf{w}_{2} .
\end{aligned}
$$

In these equations $\boldsymbol{\Gamma}_{11}$ and $\boldsymbol{\Gamma}_{22}$ are the submatrices of $\boldsymbol{\Gamma}$ corresponding to the first and second subset, whereas $\boldsymbol{\Gamma}_{12}$ corresponds to the coherence between the two subsets.

This last matrix $\boldsymbol{\Gamma}_{12}$ will be available only if one can operate on the two subsets at the same time, so that this kind of optimization might not be always possible. One example would be the use of stacking to "compress" a year of acquisitions in a multi-year SAR satellite mission, for which storing all focused images would be not feasible.

\section{EXPERIMENTS WITH TERRASAR-X}

We apply the proposed procedure to a stack of 30 TerraSAR$\mathrm{X}$ images acquired between 2008 and 2009 in the region of Crotone, Italy. The images have been coregistered to a common reference and a topographic phase component has been removed (SRTM). Two subsets have been selected, one comprising 15 and the other 8 images. Figure 1 shows a detail of the scene.

The synthesis of the two coherent images has been done according to (5) after having estimated the phases for each subset with the algorithm found in [1] and the coherence 


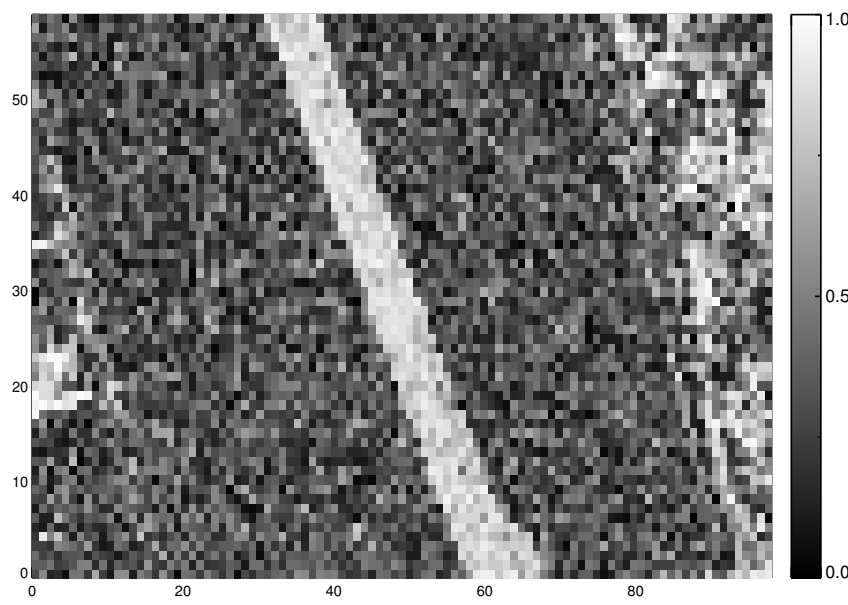

Fig. 2. Coherence between virtual images. On the airport runway the coherence is about 0.9 , which is a value not attained by any interferogram between the subsets. The coherence is computed on $5 \times 5$ windows, whereas the phases for the synthesis are computed on 10x10 windows.

magnitudes from the data themselves. The coherence between the virtual images is about 0.9 on the airport runway (Fig. 2), which is a value not attained by any pair between the two subsets. The best coherences between the two subsets are around 0.6 , and only in a few interferograms. With some additional computational effort, solving the pair of eigenvalue problems (12) and (13) allows to obtain even higher coherences.

This experiment shows the possibility to synthetize images with better interferometric capabilities compared to the original ones. The interferometric phase measured directly between two arbitrary images (2008-06-08 and 2009-12-21) is displayed in Fig. 3, whereas Fig. 4 (left) shows the same phase reconstructed with the proposed procedure.

The output of the proposed estimator is similar to the Maximum Likelihood estimator that uses all the interferograms, as Fig. 4 shows. Actually, there seem to be an additional filtering effect on the phases which is believed to be linked to the use of two window sizes, one for the estimation of the phases for the re-phasement $(10 \times 10)$ and one for the estimation of coherences $(5 \times 5)$.

\section{CONCLUSIONS}

We have proposed a new processing algorithm for stacks of SAR images, suited to scenarios in which some components of a distributed scatterer exhibit long-term coherence. This approach could be interesting for relatively long wavelength SAR missions (L-band) with short repat-pass time, if some kind of smart interferometric processing is required due to the large amount of acquired data.

Since the proposed algorithm is only suited to a particular coherence scenario, for a systematic interferometric processing one could adopt a dual approach. On one side one would do a safe processing by stacking the most coherent interferograms, on the other one would attempt to retrieve the long term coherent component, in order to achieve the best performance.

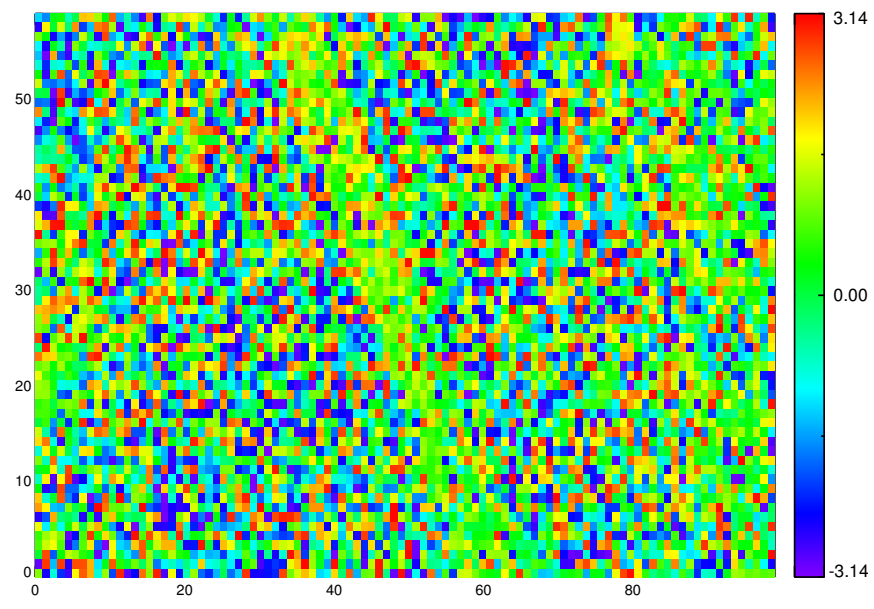

Fig. 3. The interferometric phase between two images (2008-06-08 and 2009-12-21) from direct multilooking of the interferogram.

For SARs working at shorter wavelengths (C-band, Xband) it is expected that natural targets will typically not have the desired long-term stability. However more studies are needed to characterize the long-term behavior of coherence for different terrain types and for different frequencies.

\section{ACKNOWLEDGEMENT}

The authors would like to thank the TerraSAR-X science coordinator for providing the data used in this paper and Dr. Pau Prats for the interferometric routines. 

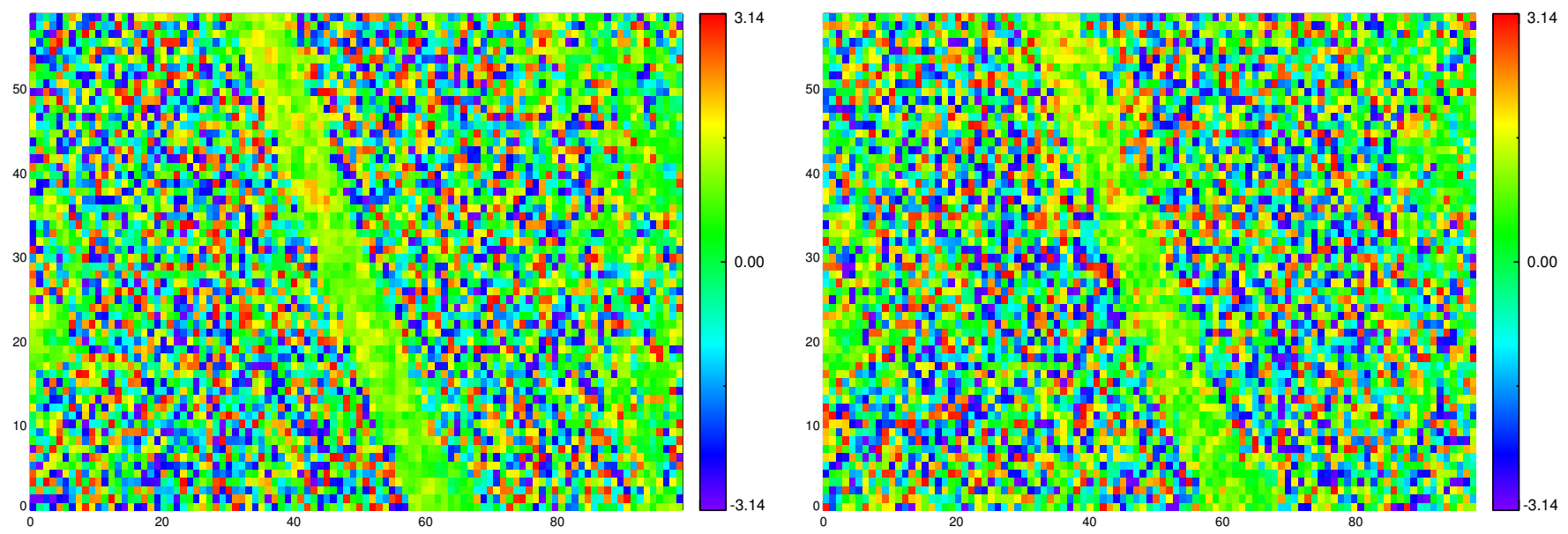

Fig. 4. Left: the interferometric phases between two images (2008-06-08 and 2009-12-21), estimated with the proposed procedure, on 5x5 pixel windows. Right: the same interferometric phases estimated with the optimum estimator (Maximum Likelihood). The two figures are not directly comparable since the phases for the re-phasement are estimated on windows of 10x10 (left) which indirectly seems to yield a filtering effect on the final phase.

\section{REFERENCES}

[1] A. Monti Guarnieri and S. Tebaldini, "On the exploitation of target statistics for SAR interferometry applications," IEEE Trans. Geosci. Remote Sensing, vol. 46, pp. 3436-3443, Nov 2008.

[2] A. Monti Guarnieri and S. Tebaldini, "Hybrid Cramér-Rao bound for crustal displacement field estimators in SAR interferometry," IEEE Signal Proc. Letters, vol. 14, pp. 1012-1015, Dec 2007.

[3] R. Lanari, F. Casu, M. Manzo, G. Zeni, P. Berardino, M. Manunta, and A. Pepe, "An overview of the Small BAseline Subset algorithm: a DInSAR technique for surface deformation analysis," Pure and Applied Geophysics, vol. 164, no. 4, pp. 637-661, Apr 2007.

[4] F. De Zan, P. Prats, and G. Krieger, "Mission design and peformance for systematic deformation measurements with a spaceborne SAR system," IGARSS 2009, Proceedings of, Nov 2009.

[5] A. Ferretti, F. Novali, F. De Zan, C. Prati, and F. Rocca, "Moving from PS to slowly decorrelating targets: A prospective view," Proc. of EUSAR $2008,2008$.

[6] A. Kavčić and J. Moura, "Matrices with banded inverses: inversion algorithms and factorization of Gauss-Markov processes," Information Theory, IEEE transactions on, vol. 46, no. 4, pp. 1495-1509, Jul 2000.

[7] M. Shimada, A. Rosenqvist, M. Watanabe, and T. Tadono, "The polarimetric and interferometric potential of ALOS PALSAR," Proc. of POLinSAR 2005 Workshop, 2005.

[8] A. Parizzi, X. Y. Cong, and M. Eineder, "First results from multifrequency interferometry - a comparison of different decorrelation time constants at X, C and L-band," Fringe 2009 Workshop Proceedings, 2009.

[9] F. De Zan, "Interferometric performance aspects for Tandem-L," Proc. of Fringe 2009 Workshop, pp. 1-5, 2010.

[10] R. Bamler and M. Eineder, "Accuracy of differential shift estimation by correlation and split-bandwidth interferometry for wideband and deltak SAR systems," IEEE Geosci. Remote Sensing Letters, vol. 2, pp. 151-155, Apr 2005.

[11] A. Ferretti, C. Prati, and F. Rocca, "Permanent scatterers in SAR interferometry," Geoscience and Remote Sensing, IEEE Transactions on, vol. 39, no. 1, pp. 8-20, Jan 2001.

[12] S. Cloude and K. Papathanassiou, "Polarimetric SAR interferometry" Geoscience and Remote Sensing, IEEE Transactions on, vol. 36, no. 5, pp. 1551-1565, Sept 1998.

[13] M. Neumann, Ferro-Famil L., and A. Reigber, "Multibaseline polarimetric SAR interferometry coherence optimization," Geoscience and Remote Sensing Letters, IEEE, vol. 5, no. 1, pp. 93-97, Jan 2008. 